\title{
Correction: S100A9+ MDSC and TAM-mediated EGFR-TKI resistance in lung adenocarcinoma: the role of RELB
}

\author{
Po-Hao Feng ${ }^{1,2}$, Chih-Teng $\mathbf{Y u}^{3}$, Kuan-Yuan Chen ${ }^{1,4}$, Ching-Shan Luo ${ }^{1}$, Shen Ming \\ $\mathbf{W u}^{1}$, Chien-Ying Liu ${ }^{3}$, Lu Wei Kuo ${ }^{1}$, Yao-Fei Chan ${ }^{3}$, Tzu-Tao Chen ${ }^{1}$, Chih-Cheng

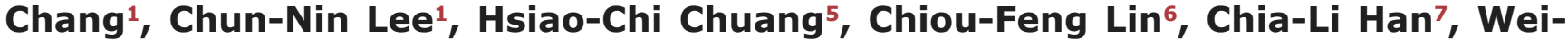 \\ Hwa Lee ${ }^{8}$ and Kang-Yun Lee ${ }^{1,2}$ \\ ${ }^{1}$ Division of Pulmonary Medicine, Department of Internal Medicine, Shuang Ho Hospital, Taipei Medical University, Taipei, \\ Taiwan \\ ${ }^{2}$ Division of Thoracic Medicine, Department of Internal Medicine, School of Medicine, College of Medicine, Taipei Medical \\ University, Taipei, Taiwan \\ ${ }^{3}$ Division of Pulmonary Medicine, Department of Internal Medicine, Chang Gung Medical Foundation Linko Branch, Taoyuan, \\ Taiwan \\ ${ }^{4}$ Graduate Institute of Clinical Medicine, College of Medicine, Taipei Medical University, Taipei, Taiwan \\ ${ }^{5}$ School of Respiratory Therapy, College of Medicine, Taipei Medical University, Taipei, Taiwan \\ ${ }^{6}$ Department of Microbiology and Immunology, School of Medicine, College of Medicine, Taipei Medical University, Taipei, \\ Taiwan \\ ${ }^{7}$ Master Program for Clinical Pharmacogenomics and Pharmacoproteomics, College of Pharmacy, Taipei Medical University, \\ Taipei, Taiwan \\ ${ }^{8}$ Department of Pathology, Shuang Ho Hospital, Taipei Medical University, Taipei, Taiwan \\ Published: July 31, 2018
}

Copyright: Feng et al. This is an open-access article distributed under the terms of the Creative Commons Attribution License 3.0 (CC BY 3.0), which permits unrestricted use, distribution, and reproduction in any medium, provided the original author and source are credited.

This article has been corrected: The correct funding information is listed below:

This study was supported by National Science Council (NSC 102-2314-B-038-048) to PHF, Ministry of Science and Technology (MOST 103-2314-B-038 -056 and 104-2314-B-038-070) to PHF, and Taipei Medical University (TMU103AE1-B31, TMU103-AE2-I01-3, 103TMU-SHH-01-2, 105TMU-SHH-02-2) to PHF.

Original article: Oncotarget. 2018; 9:7631-7643. https://doi.org/10.18632/oncotarget.24146 